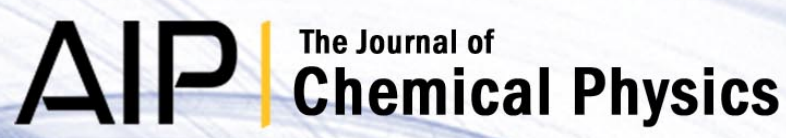

\section{Excess protons in water-acetone mixtures. II. A conductivity study}

Rocío Semino and M. Paula Longinotti

Citation: J. Chem. Phys. 139, 164510 (2013); doi: 10.1063/1.4826464

View online: http://dx.doi.org/10.1063/1.4826464

View Table of Contents: http://jcp.aip.org/resource/1/JCPSA6/v139/i16

Published by the AIP Publishing LLC.

Additional information on J. Chem. Phys.

Journal Homepage: http://jcp.aip.org/

Journal Information: http://jcp.aip.org/about/about_the_journal

Top downloads: http://jcp.aip.org/features/most_downloaded

Information for Authors: http://jcp.aip.org/authors

\section{AlP Re-register for Table of Content Alerts}

\section{Create a profile.




\section{Excess protons in water-acetone mixtures. II. A conductivity study}

Rocío Semino and M. Paula Longinottia)

Departamento de Química Inorgánica, Analíica y Química Física (DQIAQF)/Instituto de Química Física de los Materiales, Medio Ambiente y Energía (INQUIMAE-CONICET), Facultad de Ciencias Exactas y

Naturales, Universidad de Buenos Aires, Pabellón II, Ciudad Universitaria, (1428), Buenos Aires, Argentina

(Received 7 August 2013; accepted 6 October 2013; published online 28 October 2013)

In the present work we complement a previous simulation study [R. Semino and D. Laria, J. Chem. Phys. 136, 194503 (2012)] on the disruption of the proton transfer mechanism in water by the addition of an aprotic solvent, such as acetone. We provide experimental measurements of the mobility of protons in aqueous-acetone mixtures in a wide composition range, for water molar fractions, $x_{w}$, between 0.05 and 1.00. Furthermore, new molecular dynamics simulation results are presented for rich acetone mixtures, which provide further insight into the proton transport mechanism in water-nonprotic solvent mixtures. The proton mobility was analyzed between $x_{w} 0.05$ and 1.00 and compared to molecular dynamics simulation data. Results show two qualitative changes in the proton transport composition dependence at $x_{w} \sim 0.25$ and 0.8. At $x_{w}<0.25$ the ratio of the infinite dilution molar conductivities of $\mathrm{HCl}$ and $\mathrm{LiCl}, \Lambda^{0} \mathrm{HCl} \cdot \Lambda^{0} \mathrm{LiCl}^{-1}$, is approximately constant and equal to one, since the proton diffusion is vehicular and equal to that of $\mathrm{Li}^{+}$. At $x_{w} \sim 0.25$, proton mobility starts to differ from that of $\mathrm{Li}^{+}$indicating that above this concentration the Grotthuss transport mechanism starts to be possible. Molecular dynamics simulation results showed that at this threshold concentration the probability of interconversion between two Eigen structures starts to be non-negligible. At $x_{w} \sim 0.8$, the infinite molar conductivity of $\mathrm{HCl}$ concentration dependence qualitatively changes. This result is in excellent agreement with the analysis presented in the previous simulation work and it has been ascribed to the interchange of water and acetone molecules in the second solvation shell of the hydronium ion. (C) 2013 AIP Publishing LLC. [http://dx.doi.org/10.1063/1.4826464]

\section{INTRODUCTION}

Aqueous protons play an important role in many chemical and biological reactions as well as in technological devices. ${ }^{2}$ Despite its seemingly simple nature, proton transport has proven to be a complex process. The ionic molar conductivity at infinite dilution of the aqueous proton is almost ten times larger than that of a small simple cation such as $\mathrm{Li}^{+}$. It is generally accepted that, in addition to the usual Fickian behavior, aqueous proton exhibits a transport mechanism known as Grotthuss mechanism, ${ }^{3}$ where proton is depicted as a fluxional defect along the hydrogen bond network. Proton transfer occurs in the picoseconds timescale, which is the same as the one for hydrogen bonds formation and cleavage dynamics. ${ }^{4}$ From the structural perspective, proton can be regarded as adopting a series of continuous structures intermediate between the resonant Zundel dimer ${ }^{5} \mathrm{H}_{5} \mathrm{O}_{2}{ }^{+}$and the Eigen tetramer ${ }^{6} \mathrm{H}_{9} \mathrm{O}_{4}{ }^{+}$, an hydronium, $\mathrm{H}_{3} \mathrm{O}^{+}$, strongly solvated by three water molecules. The interconversion energy between both species has been measured, ${ }^{7}$ as well as calculated by molecular dynamics simulations ${ }^{8}$ and it has been found that the Eigen is $\sim 1-2 \mathrm{kcal}$ more stable than the Zundel. It has been suggested that the proton transfer mechanism can be interpreted as a conversion from an Eigen centered in a water molecule into a new Eigen centered in another water molecule. The intermediate structure between the two Eigen

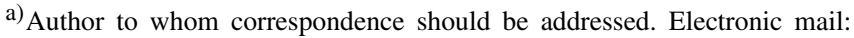
longinot@qi.fcen.uba.ar
}

complexes would correspond to a Zundel-like complex, comprising the acceptor and donor molecules. ${ }^{9}$

The changes that arise in proton mobility when adding increasing amounts of an organic solvent to a diluted acid aqueous solution have been extensively studied, , ${ }^{1,10-18}$ since they provide valuable insights into the interrelation between hydrogen bonds dynamics and proton diffusion. The organic solvents selected for this analysis should be fully miscible with water over a wide range of compositions. Within these solvents, the protic ones are involved in the Grotthuss mechanism while the aprotic ones are not. In the latter case, the effects of the organic solvent in the proton transport mechanism appear to be merely geometrical.

Many studies addressing this issue have been carried out, covering both theoretical and experimental techniques. From the experimental perspective, electrical conductivity measurements have been widely employed with the aim of disclosing the nature of proton mobility. From the theoretical perspective, a considerable amount of work has been undertaken in order to develop fast and reliable methods to study proton transport.

In the water-protic solvent case, Goffredi and Shedlovsky ${ }^{10}$ have studied the conductivity of $\mathrm{HCl}$ and $\mathrm{NaCl}$ as a function of composition in water - 1-propanol mixtures. Their measurements show that the Grotthuss proton conductivity is present in mixtures with $x_{w} \geq 0.45$. For smaller $x_{w}$, proton conductivity becomes similar to that of $\mathrm{Na}^{+}$, and finally in alcohol rich solutions it starts to rise again, since the organic solvent in this concentration region is 
also involved in a Grotthuss-like mechanism. This system has also been covered in an extensive study based on conductivity measurements of $\mathrm{HCl}$ in water mixed with many different organic solvents. ${ }^{11}$ Furthermore, methanol-water mixtures have been studied with molecular dynamics techniques ${ }^{12}$ based on a valence bond formalism (MS-EVB ${ }^{8}$ ) as well as with more accurate but more computationally expensive $a b$ initio molecular dynamics techniques. ${ }^{13}$ All these previous works provide enough information to conclude that there is a gradual deactivation of the Grotthuss mechanism when the alcohol content in the mixture is increased, up to a certain composition where there is evidence of contribution from a Grotthuss-like mechanism involving hydrogen bonding between alcohol-alcohol and alcohol-water molecules. The magnitude of this effect increases with decreasing size of the alcohol hydrocarbon chain.

In the water-aprotic solvent mixtures group, Gileadi and collaborators ${ }^{14,15}$ have studied proton conductivity in acetonitrile-water and tetrahydrofuran-water mixtures. The authors analyze the ratio between $\mathrm{HClO}_{4}$ and $\mathrm{LiClO}_{4}$ conductivities at infinite dilution. They find that this ratio is constant and practically equal to one up to a water molar fraction $x_{w} \sim 0.25$, implying that the Grotthuss mechanism does not contribute to proton transport in the aprotic solvent rich solutions. This result is independent of the aprotic solvent in the mixture. As a possible interpretation of this effect they suggest that the aprotic solvent destroys the short range order present in water solutions. In this context, the role of the organic solvent would be to geometrically hinder the proton transport by the Grotthuss mechanism, by disrupting the water hydrogen bonds network. Similar results could be inferred for sulfolane-water ${ }^{16}$ and ethylene carbonate-water mixtures, ${ }^{17}$ although no measurements were performed in these studies for aprotic solvent rich solutions. Conductivity measurements of $\mathrm{HCl}$ in water-acetone mixtures were carried out in a previous work dating back to $1925 .{ }^{18}$ However, this study does not span adequately the whole interval of compositions to allow for a comprehensive interpretation of the proton transport in these mixtures. Moreover, the experimental measurements as well as the method employed for analyzing the data were not as accurate as the ones available nowadays.

In Paper I, ${ }^{1}$ proton structure and dynamics in acetonewater mixtures have been analyzed by molecular dynamics techniques, employing the MS-EVB approach. ${ }^{8}$ It has been found that proton diffusion coefficient increases with composition above $x_{w}=0.25$ with a qualitative change around $x_{w}$ $\sim 0.8$ where the proton transfer rate also changes. For mixtures with $0.25<x_{w}<0.8$, the proton transfer mechanism was found to be controlled by the presence of acetone molecules in the local vicinity of the Eigen. The authors proposed a three stage mechanism: first, for the proton translocation process to begin, an acetone molecule in the second solvation shell of the hydronium must be replaced by one of water. Then, there is a period of rapid proton resonance between the two water molecules associated with the former hydronium and the new one (the structure of the proton can be regarded as that of a Zundel dimer at this stage). Finally, there must be a new interchange around the former hydronium in which an acetone molecule must replace one of water in its hydrogen bond acceptor role. This last step inhibits the resonance and the proton is stabilized in the new Eigen structure.

In this work we present conductivity measurements of $\mathrm{HCl}$ and $\mathrm{LiCl}$ in water-acetone mixtures in a wide composition range and new molecular dynamics simulations data, which provide further insights into the molecular interpretation of proton transport in these mixtures. The conclusions derived from this analysis complement and are in excellent agreement with the above mentioned previous studies. ${ }^{1,14,15}$

This paper is organized as follows. Section II is subdivided in three subsections. In Sec. II A, experimental details of the conductivity measurements are provided, in Sec. II B, the model with which conductivity results were analyzed is detailed and in Sec. II C there is a brief explanation of the molecular dynamics method employed. In Sec. III conductivity and simulation results are presented and compared with results taken from other studies. Finally, the conclusions are summarized in Sec. IV.

\section{METHODS}

\section{A. Conductivity measurements}

Acetone (Merck p.a., > 99\%), milliq water, anhydrous $\mathrm{LiCl}$ (Merck, p.a.), and $\mathrm{HCl}$ (37\%, Anedra) were used as received. The hydration of the organic solvent was not determined, considering that for the composition range analyzed in this work the water content is negligible with respect to the amount of water added to prepare the mixtures.

A conductivity cell with a mixing bulb and platinized platinum electrodes, previously employed for high precision measurements, ${ }^{19,20}$ was used to determine the conductivity of $\mathrm{HCl}$ and $\mathrm{LiCl}$ in aqueous acetone mixtures as a function of electrolyte concentration $\left(1 \times 10^{-5}-7 \times 10^{-3} \mathrm{M}\right)$ and solvent composition $\left(x_{w}=0.05-1.00\right)$. The cell constant, $k_{\text {cell }}$ $=0.01003 \pm 0.00003 \mathrm{~cm}^{-1}$ at $298.15 \mathrm{~K}$, was determined using a standard $\mathrm{KCl}$ aqueous solution of known specific conductivity. ${ }^{21}$

The conductivity measurements were performed according to the following protocol: first, the solvent was added to the conductivity cell by weighting the corresponding amounts of acetone and water to obtain the desired compositions. Then, the cell was thermostatized in an oil bath at 298.15 $\pm 0.04 \mathrm{~K}$; after reaching thermal equilibrium, the solvent resistance was measured. Afterwards, increasing weighted amounts of a stock solution of $\mathrm{LiCl}$ or $\mathrm{HCl}$, prepared in a water-acetone mixture of equal composition, were added. Additions were carried out under $\mathrm{N}_{2}$ atmosphere only for water rich solutions $\left(x_{w}>0.5\right)$ since for acetone rich mixtures the $\mathrm{N}_{2}$ bubbling would lead to evaporation of acetone and consequently, to changes in the composition of the mixture. Thus, the error introduced would be larger than that induced by $\mathrm{CO}_{2}$ dissolution. The resulting solution was homogenized by manual stirring; after reaching a new thermal equilibrium, the solution resistance was determined. The resistance was recorded at several frequencies between 0.2 and $7.5 \mathrm{kHz}$ and the extrapolated value at infinite frequency was computed.

The $\mathrm{LiCl}$ stock solution was prepared by weighting known amounts of acetone, water and $\mathrm{LiCl}$, previously dried 
overnight in a vacuum heater at $393 \mathrm{~K}$. For solutions with $x_{w}<0.25, \mathrm{LiCl}$ dissolution required mixture sonication. The $\mathrm{HCl}$ stock solution was prepared by weighting known amounts of acetone and $0.482 \pm 0.006 \mathrm{~mol} \mathrm{~kg} \mathrm{~kg}^{-1} \mathrm{HCl}$ aqueous stock solution. The acid concentration in the aqueous stock solution was determined by duplicate by neutralizing a weighted amount of dried $\mathrm{Na}_{2} \mathrm{CO}_{3}$.

The acid and salt molar conductivities, $\Lambda$, were determined according to

$$
\Lambda=\frac{\kappa}{c}=\left(\frac{1}{R}-\frac{1}{R_{0}}\right) \frac{k_{c e l l}}{c},
$$

where $\kappa$ is the solution specific conductivity, $c$ is the electrolyte molar concentration, $R$ is the solution resistance, and $R_{O}$ is the solvent resistance.

For the resistance measurements a Precise LCR meter $\left(\mathrm{G}^{\mathrm{w}} \mathrm{INSTEK}\right)$ was used. An AC voltage $(500 \mathrm{mV})$ was applied to the electrodes at different frequencies and the resistive and capacitive components were recorded, considering an equivalent resistance-capacitance parallel circuit.

\section{B. Data analysis}

The molar conductivity of electrolytes in solvents of moderate dielectric constants decreases with increasing concentration due to ion-solvent effects and ionic association, the latter described by an association constant $K_{a}$. One of the goals of this work is to obtain this parameter and the molar conductivity extrapolated at infinite dilution, $\Lambda^{0}$, since they provide relevant physical information of the system. For this purpose, the concentration dependence of the molar conductivity of $\mathrm{LiCl}$ and $\mathrm{HCl}$ was analyzed using the following Fuoss-Krauss equation, ${ }^{22}$

$$
\frac{T(z)}{\Lambda}=\frac{1}{\Lambda^{0}}+\left(\frac{c \gamma_{ \pm}^{2} \Lambda}{T(z)}\right)\left(\frac{K_{a}}{\left(\Lambda^{0}\right)^{2}}\right) .
$$

In Eq. (2) $T(z)=1-z\left[1-z(1-\ldots)^{-1 / 2}\right]^{-1 / 2} \approx(1-z)$ with $z=S(\Lambda c)^{1 / 2}\left(\Lambda^{0}\right)^{-3 / 2} . S$ is the limiting Onsager's law slope, which depends on the charge of the electrolyte, the temperature and the viscosity and dielectric constant of the solvent. $\gamma_{ \pm}$is the mean activity coefficient which was approximated by the Debye-Hückel equation,

$$
\ln \gamma_{ \pm}=-\frac{A(\alpha c)^{1 / 2}}{1+B q(\alpha c)^{1 / 2}}
$$

where $\alpha$, the dissociation degree, is given by

$$
\alpha=\frac{\Lambda}{\Lambda^{0} T(z)} .
$$

$A$ and $B$ are the Debye-Hückel constants which depend on the temperature and solvent properties, such as the density and the dielectric constant. $q$ is the Bjerrum distance which depends on the charge of the electrolyte, the temperature, and the dielectric constant of the solvent.

$\Lambda^{0}$ and $K_{a}$ were calculated by a self consistent procedure using Eq. (2) by plotting $T(z) \Lambda^{-1} v s . c \gamma_{ \pm}^{2} \Lambda T(z)^{-1}$. An initial guess value of $\Lambda^{0}$ was given to calculate a new value of $\Lambda^{0}$ and $K_{a}$ from the slope and intercept of the previously mentioned linear regression. The obtained $\Lambda^{0}$ was used as an input value in the following linear fit and this procedure was repeated until convergence was reached. The convergence criterion was established considering a difference between the input and the resulting $\Lambda^{0}$ values smaller than the error obtained for this parameter from the linear fit.

One could eventually think of more refined conductivity equations to describe the dependence of the ionic molar conductivity on concentration for associated and non-associated electrolytes, such as the one proposed by Fuoss-Hsia-Fernández Prini. ${ }^{23}$ However, for the concentration range studied in this work, a simpler description of the molar conductivity concentration dependence adequately describes the conductivity data.

For aqueous acetone mixtures where electrolyte association was not observed, molar ionic conductivity data were fitted with Eq. (2) fixing $K_{a}=0$. This assumption gives an estimation of $\Lambda^{0}$ with an error of approximately $1 \%-2 \%$. This error was estimated determining the difference between the calculated $\Lambda^{0}$ and the $\Lambda^{0}$ values published in the literature for $\mathrm{LiCl}$ and $\mathrm{HCl}$ in pure water. ${ }^{24}$ Considering the dependence of $\Lambda^{0}$ of both electrolytes on solvent composition, this error is small enough to sustain the analysis performed in this work.

Systematic deviations were observed when Eq. (2) was used to fit the conductivity data of $\mathrm{HCl}$ in aqueous acetone mixtures with $x_{w} \leq 0.10$. This behavior could be ascribed to the formation of ionic triplets, as already observed in conductivity measurements of $\mathrm{HCl}$ in pure sulfolane. ${ }^{16}$ Therefore, the data were analyzed by using the expression by FuossKrauss $^{22}$ that includes effects derived from the presence of ionic triplets:

$$
\Lambda g(c) c^{1 / 2}=\frac{\Lambda^{0}}{K_{a}^{1 / 2}}+\frac{\Lambda_{T}^{0} K_{T}}{K_{a}^{1 / 2}}\left(1-\frac{\Lambda}{\Lambda^{0}}\right) c,
$$

where

$$
g(c)=\frac{\gamma_{ \pm}}{(1-z)\left(1-\Lambda / \Lambda^{0}\right)^{1 / 2}} .
$$

$K_{T}$ is the formation constant of ionic triplets and $\Lambda_{T}{ }^{0}$ the molar conductivity at infinite dilution of these ions.

This equation has four unknown values, $\Lambda^{0}, K_{a}, \Lambda_{T}{ }^{0}$, and $K_{T}$. It is usually considered that $\Lambda_{T}^{0}=2 / 3 \Lambda^{0}$. Thus, there are two possible procedures for obtaining the remaining three values. One is to assume the validity of the Walden Rule $\left(\Lambda^{0} \eta\right.$ $=$ constant) for calculating $\Lambda^{0}$ as a function of water-acetone composition using data in pure acetone or pure water. However, it is well known that the relationship between the infinite dilution molar ionic conductivity and the solvent viscosity is constant only for ions whose diffusion is vehicular and whose size is comparable or bigger to the solvent molecules. Thus, the Walden rule is not valid for the transport of acids in mixtures of water with organic solvents. ${ }^{14}$ Therefore, we adopted a different procedure, previously proposed by Goldfarb et al., ${ }^{25}$ to obtain $\Lambda^{0}, K_{a}$, and $K_{T}$ by Eq. (5). A value of $\Lambda^{0}$ is assumed, and $K_{a}$ and $K_{T}$ are calculated from the slope and intercept of the linear regression of $\Lambda g(c) c^{1 / 2} v s$. $\left(1-\Lambda / \Lambda^{0}\right) c$. Then, a different $\Lambda^{0}$ value is assumed and the same fit is repeated until a minimum value for the standard 
deviation of the fit is obtained. The $\Lambda^{0}$ which gives the minimum standard deviation of the fit is the chosen value.

The analysis of the conductivity data with Eq. (2) or (5) requires the knowledge of some physical properties of the solvent mixtures, such as the dielectric constant, the density, and the viscosity. Dielectric constants for acetone-water mixtures have been reported as a function of composition by Akerlof ${ }^{26}$ and by Dash and Patnaik. ${ }^{27}$ These data were fitted as a function of $x_{w}$ using a polynomial equation. Viscosity data, reported by Akerlof, ${ }^{26}$ Stairs, ${ }^{28}$ and Noda et al.,${ }^{29}$ were fitted in logarithmic scale as a function of $x_{w}$. The densities of the aqueous acetone mixtures reported by Estrada-Baltazar ${ }^{30}$ were fitted as a polynomial function of $x_{w}$. All the physical properties data of the aqueous acetone mixtures used were reported at $298.15 \mathrm{~K}$.

\section{Computer simulations}

We carried out molecular dynamics simulations of two systems comprising an excess proton in water-acetone mixtures for water molar fractions $x_{w}=0.20$ and 0.10 . Trajectories were generated using the same procedure as the one used in Ref. 1. The methodology is based on a MS-EVB hamiltonian. ${ }^{8}$ There is extensive bibliography describing this kind of methodology, so we will present here a brief summary; more details can be found at Ref. 31 .

The EVB formalism was originally proposed by Warshel and Weiss. ${ }^{32}$ The basic idea is to describe an excess proton as a linear combination of diabatic states where the proton is considered to be localized at a tagged water molecule. The time evolution of the systems is obtained as follows (for more details see Refs. 1 and 8): (i) the topology is analyzed and a finite number of diabatic states are identified in the close vicinity of the excess charge, (ii) an MS-EVB Hamiltonian is constructed, (iii) the hamiltonian matrix is diagonalized and the coefficients accounting for contributions from each diabatic state to the state of the excess proton are obtained. The identity of the diabatic state with the largest coefficient is updated and serves to identify a proton translocation event, (iv) with the potential energy surface obtained from the diagonalization, forces between the nuclei are computed by applying the Hellmann-Feynman theorem.

Since acetone has much lower proton affinity than that of water $\left(\mathrm{pKa} \mathrm{H}_{3} \mathrm{O}^{+}=-1.7\right.$ and $\left.\mathrm{pKa}\left(\mathrm{CH}_{3}\right)_{2} \mathrm{CHO}^{+}=-7.2\right)$, diabatic states where the excess proton is bonded to an acetone molecule were not considered in the EVB hamiltonian.

The simulated systems consisted of cubic boxes with full periodicity. The total number of molecules of water and acetone was 125 in all cases. The length of the box was adjusted to match the experimental densities. ${ }^{30}$ Initially, the molecules were randomly distributed. Then, equilibration $\sim 1$ ns runs, with velocity rescaling at $T=298 \mathrm{~K}$, were performed. An excess proton was added, and the system was equilibrated for $\sim 0.5 \mathrm{~ns}$. We collected statistics along microcanonical trajectories lasting $\sim 2 \mathrm{~ns}$. The typical number of diabatic states considered in each simulation step was between 3 and 6.

\section{RESULTS AND DISCUSSION}

Fig. 1 shows the values of molar conductivities at infinite dilution for $\mathrm{HCl}$ and $\mathrm{LiCl}$ as a function of the composition of the acetone-water mixtures. $\Lambda^{0}$ exhibits non-monotonic concentration dependence for both electrolytes. For $\mathrm{HCl}$, it decreases as $x_{w}$ increases, reaching a minimum value of two thirds of its value for $x_{w} \sim 0$ at $x_{w} \sim 0.4$. At higher water contents $\Lambda^{0}$ rises non-uniformly, first with an approximately constant curvature up to $x_{w} \sim 0.8$, and then much more markedly, almost triplicating its value determined for $x_{w} \sim 0$, for pure water. In the case of $\mathrm{LiCl}, \Lambda^{0}$ is minimum at $x_{w} \sim 0.8$, with a value of around of one third of its value at $x_{w} \sim 0$ and with a uniform dependence of $\Lambda^{0}$ vs $x_{w}$ along the whole composition range. We assigned the observed variation in the curvature of $\Lambda^{0}{ }_{\mathrm{HCl}}$ vs $x_{w}$ at $x_{w} \sim 0.8$ to the change in the Grotthuss mechanism above this threshold concentration, as has been proposed by Semino and Laria. ${ }^{1}$ This effect is absent for $\mathrm{LiCl}$ since the transport mechanism for $\mathrm{Li}^{+}$cation is vehicular along the whole composition range.

The viscosity of water-acetone mixtures $26,28,29$ is strongly dependent on the composition of the mixture, showing a maximum at $x_{w} \sim 0.85$. Therefore, $\Lambda^{0}{ }_{\mathrm{LiCl}} v s x_{w}$ presents a minimum value around this composition. Thus, in order to eliminate the viscosity effect in the dependence of the diffusion of the electrolyte with composition, we plotted in Fig. 2 the Walden product for $\mathrm{LiCl}$ and $\mathrm{HCl}$ as a function of composition.

For $\mathrm{LiCl}$ the Walden product presents a much milder composition dependence compared to $\mathrm{HCl}$ since the diffusion of the former electrolyte is only vehicular, while for $\mathrm{HCl}$ the Grotthuss mechanism controls the proton transport at high water contents. Note that, for $\mathrm{LiCl}$ the Walden product is not strictly constant in the whole composition range. This is due to the fact that the Walden rule cannot be applied to describe the mobility of ions of small sizes compared to the solvent molecules. The description of the molar conductivity viscosity dependence in these cases should include an additional term referred to as dielectric friction. ${ }^{33-36}$

In order to analyze the effect of the Grotthuss mechanism in the proton mobility, eliminating to the greatest extent other

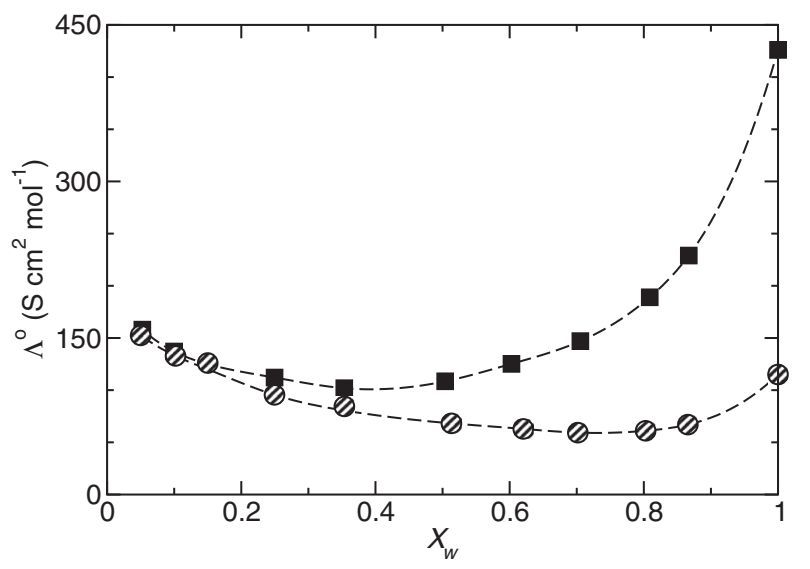

FIG. 1. Conductivity at infinite dilution for $\mathrm{HCl}$ (black squares) and $\mathrm{LiCl}$ (striped circles) as a function of water molar fraction $\left(x_{w}\right)$ for water-acetone mixtures. The dashed lines were added as a guide to the eye. 


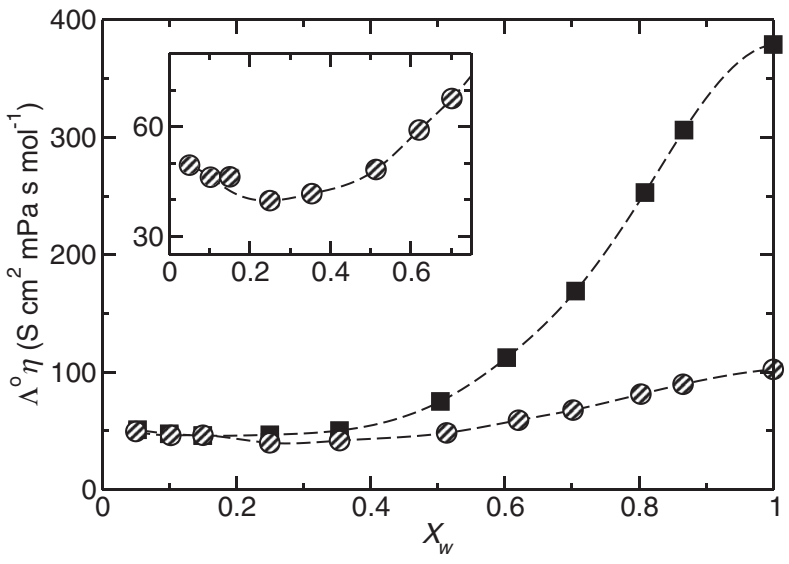

FIG. 2. Walden product for $\mathrm{HCl}$ and $\mathrm{LiCl}$ as a function of the composition $x_{w}$ of the acetone-water mixture. The symbols are the same as those used in Fig. 1. The dashed lines were added as a guide to the eye. The inset shows a zoom of the region $0<x_{w}<0.75$ for the Walden product of $\mathrm{LiCl}$.

possible effects, such as the influence of the conductivity of $\mathrm{Cl}^{-}$and the vehicular diffusion (the one controlled by the viscosity of the solvent and the only kind of diffusion in the case of $\left.\mathrm{Li}^{+}\right)$in Fig. 3 we plotted the ratio $R=\Lambda^{0}{ }_{\mathrm{HCl}}\left(\Lambda^{0}{ }_{\mathrm{LiCl}}\right)^{-1}$ as a function of the composition of the mixture. Two regions can be differentiated in this plot: (i) region $A, x_{w}<0.25$ and (ii) region $B, x_{w}>0.25$. In region $A, R$ is practically equal to one. This means that the mobility of $\mathrm{H}^{+}$is comparable to that of $\mathrm{Li}^{+}$, and therefore its diffusion is only vehicular. For these mixtures, acetone is the major component, and the Grotthuss mechanism might be blocked due to the characteristics of the hydrogen bond network in the close vicinity of the proton. An increase in $R$ is observed around $x_{w} \sim 0.25$ where the Grotthuss mechanism starts to activate. Incidentally, the $R$ value at $x_{w} \sim 0.25$ looks bigger than expected from the tendency observed at higher $x_{w}$ values. We believe that this is not an artifact; it might be due to the minimum in the Walden product of $\mathrm{LiCl}$ at this composition as can be seen in the inset of Fig. 2.

In order to be able to provide a molecular interpretation for the breaking point at the boundary between regions $A$ and $B$, we performed molecular dynamics simulations in this con-

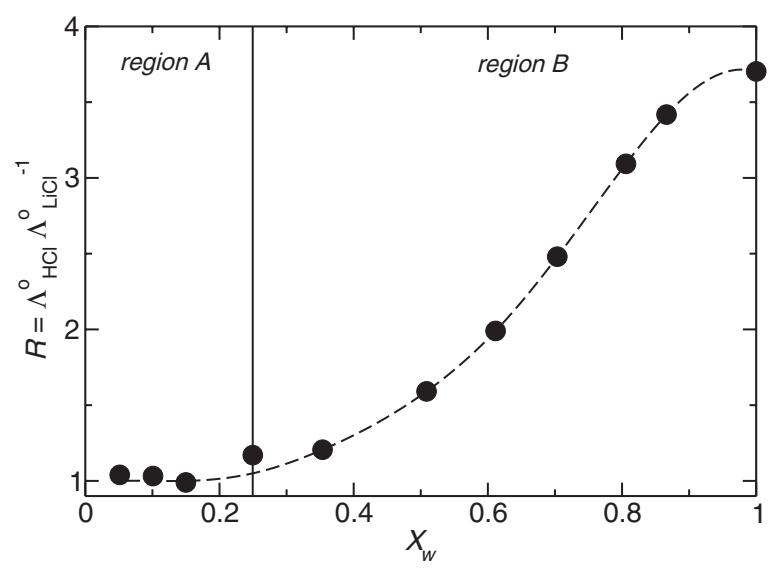

FIG. 3. $R=\Lambda^{0}{ }_{\mathrm{HCl}}\left(\Lambda^{0} \mathrm{LiCl}\right)^{-1}$ as a function of the composition $x_{w}$ of the water-acetone mixture. The dashed line was added as a guide to the eye.
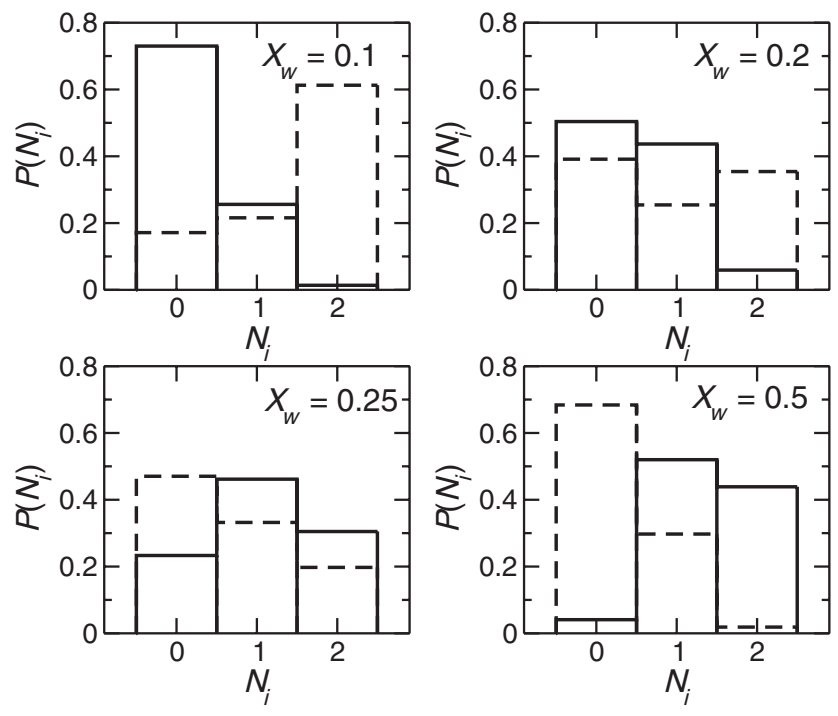

FIG. 4. Probability of finding $N_{w}$ water molecules (solid lines) and $N_{a}$ acetone molecules (dashed lines) as hydrogen bond acceptors of a tagged water molecule belonging to the first solvation shell of the hydronium, as a function of $N_{i}(i=w$ or $a)$. The upper panels show results for the simulations performed in this work, while the lower ones show results from simulations performed in Ref. 1.

centration range, since in the previous work simulation results were reported for $x_{w} \geq 0.25$. According to Semino and Laria results, for the proton transfer to occur, proton must undergo a structure of a fully hydrated Zundel, in order to allow the formation of the more stable Eigen structure. ${ }^{1}$ This implies that at least one out of the three water molecules that conform the first solvation shell of the Eigen-like hydronium, must have two water molecules acting as hydrogen bond acceptors for the translocation to be possible (these two water molecules would be part of the second solvation shell of the hydronium). In order to analyze if the fully hydrated Zundel occurs only for $x_{w}>0.25$, we found instructive to consider the probability $P\left(N_{i}\right)$ of finding $N_{i}$ molecules with $i=w$ for water and $i=a$ for acetone acting as hydrogen bond acceptors for a tagged water molecule of the first solvation shell of the hydronium. Assuming that one hydrogen could only be part of a single hydrogen bond, $N_{i}$ can adopt the values 0,1 , and 2 . As such, the fully hydrated Zundel structure requires $N_{w}=2$. In Fig. 4 we present four panels, each of them corresponds to histograms for $N_{i}$ for different mixtures. Note that $P\left(N_{w}\right)$ is practically but not exactly complementary to $P\left(N_{a}\right)$, due to a small number of configurations in which one or both of the acceptor sites are vacant. In the upper panels of Fig. 4, where mixtures in region $A$ are plotted, one can see that the probability of having a fully hydrated Zundel is less than 0.1. For $x_{w}=0.1$, the most probable scenario is $N_{w}=0$ and $N_{a}=2$, which corresponds to a fully acetone solvated Eigen. Under these circumstances, the only channel for proton transfer would involve an intermediate complex where two acetone molecules are part of the first solvation shell of the new hydronium (see Fig. 5(a)); clearly, this situation is energetically not favorable. For $x_{w}=0.2$, it is almost equally probable to have $N_{w}=0$ or 1 which, in turn, would lead to a new hydronium moiety solvated by two water molecules and one acetone if the proton translocation were to occur (see 
(a) $x_{w}=0.1$

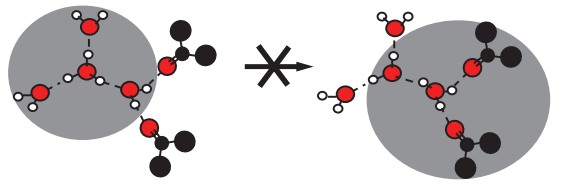

(b) $X_{w}=0.2$

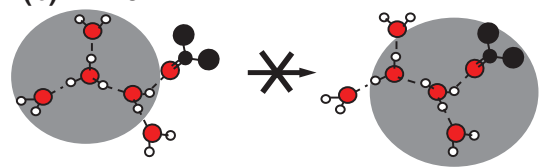

(c) $X_{w}>0.25$

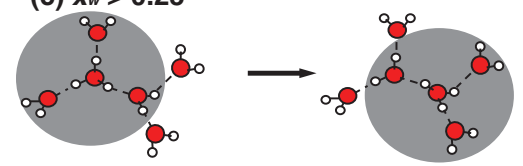

FIG. 5. Schemes of proton translocation for typical molecular topologies for (a) $x_{w}=0.1$, (b) $x_{w}=0.2$, and (c) $x_{w}>0.25$ solutions. Red circles correspond to the oxygen atoms in water and acetone molecules. White circles symbolize hydrogen atoms in water molecules and the hydronium ion. Black circles correspond to the central carbon atom and the surrounding methyl groups in acetone molecules.

Fig. 5(b)). This structure is seldom observed. The bottom left panel shows results for the limiting $x_{w}=0.25$ mixture. In this case there is a moderate probability $P\left(N_{w}\right) \sim 0.3$ for $N_{w}=2$. This probability gets even larger in the $x_{w}=0.5$ mixture (bottom right panel in Fig. 4); in this case, $N_{w}=1$ or 2 are equally probable, so upon a translocation event there is $\sim 50 \%$ chance to form a fully hydrated Zundel (see Fig. 5(c)). The picture that emerges from this observations suggests that the number of water molecules surrounding the hydronium does indeed play a crucial role in the mechanism of proton mobility by allowing the formation of a fully hydrated Zundel, as a controlling step for the proton translocation, and this seems to be possible only in mixtures with $x_{w}>0.25$ (region $B$ ).

At $x_{w} \sim 0.87$ there seems to be a change in the slope of the composition dependence of $R$ which cannot be ascribed to the variation of the proton transport mechanism around $x_{w} \sim 0.80$ observed in Fig. 1 and discussed by Semino and Laria. ${ }^{1}$ At $x_{w} \sim 0.85$ there is a maximum in the viscosity of the water-acetone mixtures which affects the diffusion of $\mathrm{Li}^{+}$ions but not the corresponding of protons. Therefore, the value of $R$ does not only depend on the Grotthuss mobility but is also influenced by the viscosity composition dependence. This can also be observed in Fig. 2 for the Walden product of $\mathrm{HCl}$, for which the composition tendency also changes around this composition value.

In order to compare the experimental data and the simulation results quantitatively, we plotted in Fig. 6 proton diffusion coefficients as a function of composition. Experimental diffusion coefficients were obtained using the following expression:

$$
\begin{aligned}
D_{H^{+}} & =\lambda_{H^{+}}\left(\frac{R T}{F^{2}}\right)=\left(\Lambda_{H C l}-\lambda_{C l^{-}}\right)\left(\frac{R T}{F^{2}}\right) \\
& =\left(\Lambda_{H C l}-\Lambda_{L i C l}+\lambda_{L i^{+}}\right)\left(\frac{R T}{F^{2}}\right) \\
& =\left(\Lambda_{H C l}-\Lambda_{L i C l}+0.3363 \Lambda_{L i C l}\right)\left(\frac{R T}{F^{2}}\right) .
\end{aligned}
$$

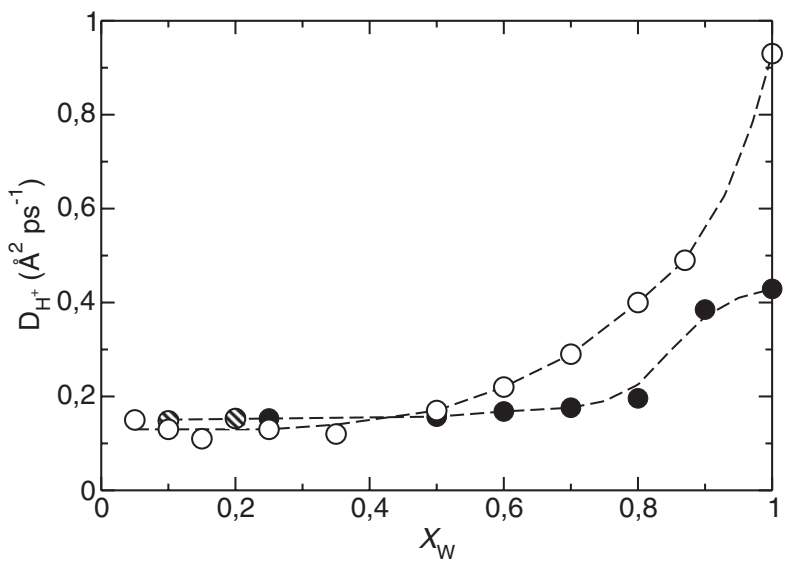

FIG. 6. Proton diffusion constants as a function of solution composition determined from experimental data as explained in the text (empty circles) and from molecular dynamics simulations (bold circles correspond to data reported in Ref. 1 and stripped circles to simulations performed in this work). The dashed lines were added as a guide to the eye.

This equation assumes the validity of the Nernst-Einstein equation and that the transport number of lithium in $\mathrm{LiCl}\left(t^{+}\right.$ $=0.3363)$ in the whole composition range is the same as that determined for its water solution.

Both series of data are similar for rich acetone mixtures but differ for water rich solutions, since quantum effects, not fully taken into account in the MSEVB method employed in our simulations, are important in these solutions (for more details see Ref. 8). It can be observed, as pointed out in Ref. 1, that the proton diffusion coefficient is practically constant at low water contents but increases above $x_{w} \sim 0.45$, where the proton transport is determined by the Grotthuss mechanism. In this case, the change in the curvature of the proton diffusion coefficient composition dependence occurs at higher $x_{w}$ values than that observed in Fig. 3 for $R$ since in the former case the data are influenced by the viscosity.

With the purpose of gaining further insight into the proton diffusion in water-aprotic solvents mixtures, in Fig. 7 we present a comparison between the results obtained in this

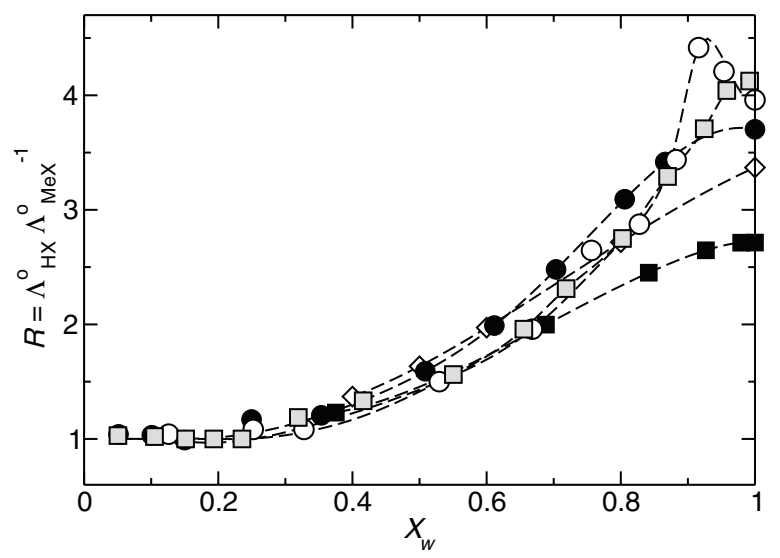

FIG. 7. $R=\Lambda^{0}{ }_{\mathrm{HX}}\left(\Lambda^{0}{ }_{\mathrm{MeX}}\right)^{-1}$, where $\mathrm{X}$ is the common anion and Me is the cation of the salt, as a function of the composition $x_{w}$ of the aprotic solvent aqueous mixture for $\mathrm{HCl} / \mathrm{LiCl}$ in acetone-water (black circles, present work), $\mathrm{HCl} / \mathrm{NaCl}$ in ethylene carbonate-water (white diamonds, Ref. 17), $\mathrm{HCl} / \mathrm{KCl}$ in sulfolane-water (black squares, Ref. 16 ), $\mathrm{HClO}_{4} / \mathrm{LiClO}_{4}$ in acetonitrilewater (grey squares, Ref. 14), $\mathrm{HClO}_{4} / \mathrm{LiClO}_{4}$ in tetrahydrofuran-water (white circles, Ref. 14). The dashed lines were added as a guide to the eye. 


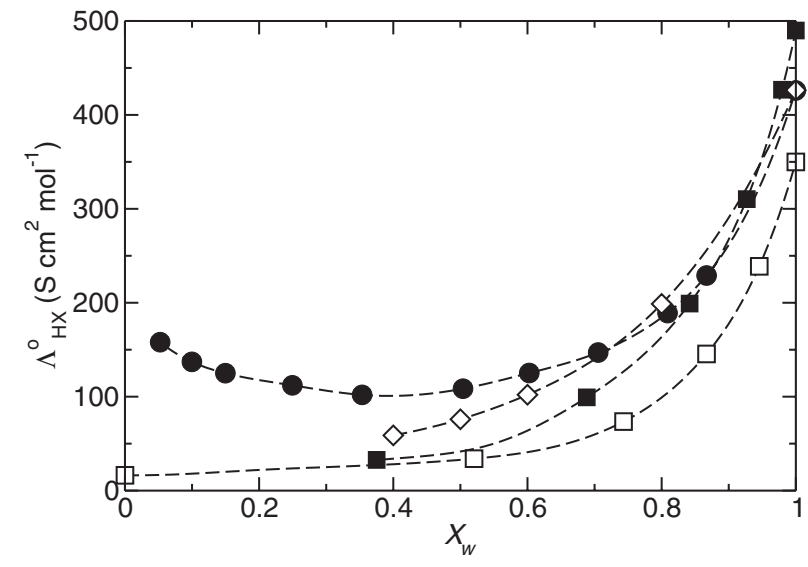

FIG. 8. $\Lambda^{0} \mathrm{HX}$, where $\mathrm{X}$ is the corresponding proton counter ion, as a function of $x_{w}$ for the same first three acids plotted in Fig. 6 and $\mathrm{HCl}$ in DMSO (white squares, Ref. 37). The dashed lines were added as a guide to the eye.

work as plotted in Fig. 3 and those reported in Refs. 14, 16, and 17. As hinted in previous works, ${ }^{14,15}$ the Grotthuss mechanism seems to start to contribute to proton transport around $x_{w} \sim 0.25$ for all systems, and this appears to be independent on the nature of the aprotic component in the mixture. This supports the idea that the effect from the aprotic solvent upon the proton mobility is purely geometrical at low water contents. Although the proton conductivities in ethylene carbonate and sulfolane water mixtures were not measured at low $x_{w}$, a similar behavior seems to be inferred from the conductivity data determined for mixtures with higher water contents. We can also observe that the composition dependence of $R$ at high water contents is determined by the nature of the aprotic solvent, since the composition and magnitude of the maximum viscosity depend on the nature of the aprotic solvent. It should be noted that, not surprisingly, the value of $R$ for pure water is different for each system, since different electrolytes were compared in the conductivity measurements.

Fig. 8 shows at high water contents a change in the curvature of $\Lambda^{0}{ }_{\mathrm{HX}}$ (with $\mathrm{X}=\mathrm{ClO}_{4}{ }^{-}$or $\mathrm{Cl}^{-}$) vs $x_{w}$ at different solvent compositions. This effect might be due to the different residence times of the aprotic solvent molecules in the second solvation shell of the hydronium and this is associated to different aprotic solvent-water intermolecular interactions.

Finally, we would like to comment on the effect of acetone on the association constants for both electrolytes. Fig. 9 shows the logarithm of $K_{a}$ as a function of the inverse of the dielectric constant. These variables are linearly correlated, as predicted by Fuoss. ${ }^{38}$ For both electrolytes, the association constants take values from $K_{a} \sim 10$ up to 6000 , getting larger as the water content is diminished, i.e., as the dielectric constant of the mixture becomes smaller. According to our results, for $x_{w}>0.5$ there is no noticeable association either for the acid or for the salt. As explained in Sec. II B, in the case of $\mathrm{HCl}$, solutions with $x_{w}=0.05$ and $x_{w}=0.10$ have been analyzed including the possibility of formation of ionic triplets. For these mixtures $\ln K_{T}=3.00$ and 1.57 for $x_{w}=0.05$ and 0.10 , respectively. In order to verify the correctness of this assumption we also measured the conductivity of $\mathrm{HCl}$ for an aqueous acetone mixture with $x_{w}=0.03$, where the influence

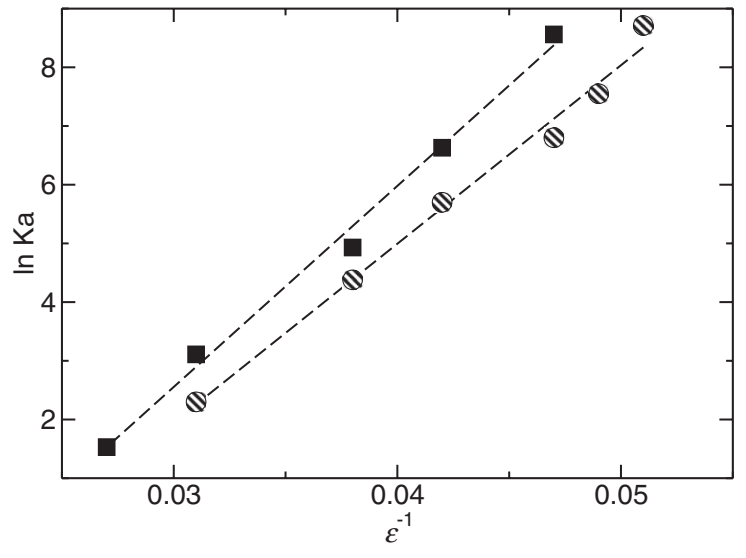

FIG. 9. Natural logarithm of the association constant $K_{a}$ as a function of the inverse of the dielectric constant $\varepsilon$ of the acetone-water mixture for $\mathrm{HCl}$ and $\mathrm{LiCl}$. The symbols are the same as those used in Fig. 1. The dashed lines were added as a guide to the eye.

of ionic triplets would be more marked. For this solution, the standard deviation of the fit of the conductivity data with Eq. (2) is exceedingly large while Eq. (5) fits the data much better, yielding $\ln K_{T}=4.70$. The presence of ionic triplets in mixtures where the aprotic solvent is predominant has already been suggested in a previous work. ${ }^{16}$ As a plausible explanation, we are lead to believe that this could be an effect of the enhancement of the local ionic density along mesoscopic water domains embedded in the aprotic solvent matrix. This enhancement could make the formation of hydrated complexes such as $\mathrm{H}_{2} \mathrm{Cl}^{+}$possible, as suggested by Voth and co-workers for concentrated hydrocloric aqueous solutions. ${ }^{39}$

\section{CONCLUSIONS}

We have performed conductivity measurements of $\mathrm{HCl}$ and $\mathrm{LiCl}$ in acetone-water mixtures in an extended composition range and estimated the proton diffusion coefficient as a function of the composition of the mixture. These results represent a complementary piece of information that bring additional support and extend the physical interpretations provided in a previous molecular dynamics study of these mixtures. ${ }^{1}$ The direct inspection of the experimental results reveals two relevant changes in the concentration dependence of the proton mobility. The first modification takes place at $x_{w} \sim 0.25$ and represents the threshold concentration beyond which the proton diffusion mechanism differs in a qualitative fashion from the one shown for a simple cation such as $\mathrm{Li}^{+}$. Results from molecular dynamics experiments show that in this concentration regime, the probability of Eigen-to-Eigen interconversion, mediated via a fully hydrated Zundel-like moiety, starts to be physically meaningful. We remark that this composition threshold has also been observed for other aprotic solvent-water mixtures. ${ }^{14,16,17,37}$

A second change in the slope of the molar conductivity of $\mathrm{HCl}$ dependence on composition at $x_{w} \sim 0.8$ was also observed confirming results from previous simulation studies that have been ascribed to interchanges of water and acetone molecules in the second solvation shells of $\mathrm{H}_{3} \mathrm{O}^{+}$. 


\section{ACKNOWLEDGMENTS}

This work has been supported by Agencia Nacional de Promoción Científica y Tecnológica (PICT 2008 No. 0628), Consejo Nacional de Investigaciones Científicas y Técnicas (CONICET PIP 095), and Universidad de Buenos Aires (Project UBACyT 20020100100519). The authors are thankful to Horacio R. Corti and Daniel Laría for fruitful discussions. M.P.L. is member of CONICET and R.S. owns a Ph.D. fellowship by CONICET.

${ }^{1}$ R. Semino and D. Laria, J. Chem. Phys. 136, 194503 (2012).

${ }^{2}$ M. H. B. Stowell, T. M. McPhillips, D. C. Rees, S. M. Soltis, E. Abresch, and G. Feher, Science 276, 812 (1997); T. Norby, Solid State Ionics 125, 1 (1999); S. J. Formosinho and L. G. Arnaut, J. Photochem. Photobiol., A 75, 21 (1993); O. A. Sineshchekov, E. G. Govorunova, J. Wang, H. Li, and J. L. Spudich, Biophys. J. 104, 807 (2013); O. Diat and G. Gebel, Nature Mater. 7, 13 (2008); B. Dong, L. Gwee, D. Salas de la Cruz, K. I. Winey, and Y. A. Elabd, Nano Lett. 10, 3785 (2010).

${ }^{3}$ C. J. T. Grotthuss, Ann. Chim. LVIII, 54 (1806).

${ }^{4}$ R. A. Robinson and R. H. Stokes, Electrolyte Solutions, 2nd ed. (Butterworths, London, 1959).

${ }^{5}$ G. Zundel and H. Metzger, Z. Phys. Chem. 58, 225 (1968).

${ }^{6}$ M. Eigen and L. de Maeyer, Proc. R. Soc. London, Ser. A 247, 505 (1958).

${ }^{7}$ Z. Luz and S. Meiboom, J. Am. Chem. Soc. 86, 4768 (1964).

${ }^{8}$ U. W. Schmitt and G. A. Voth, J. Phys. Chem. B 102, 5547 (1998); J. Chem. Phys. 111, 9361 (1999).

${ }^{9}$ O. Markovitch, H. Chen, S. Izvekov, F. Paesani, G. A. Voth, and N. Agmon, J. Phys. Chem. B 112, 9456 (2008).

${ }^{10}$ M. Goffredi and T. Shedlovsky, J. Phys. Chem. 71, 2176 (1967); 71, 2182 (1967).

${ }^{11}$ B. E. Conway, J. O'M. Bockris, and H. Linton, J. Chem. Phys. 24, 834 (1956).

${ }^{12}$ M. K. Petersen and G. A. Voth, J. Phys. Chem. B 110, 7085 (2006).

${ }^{13}$ J. A. Morrone, K. E. Haslinger, and M. E. Tuckerman, J. Phys. Chem. B 110, 3712 (2006).
${ }^{14}$ Z. Stein and E. Gileadi, J. Electrochem. Soc. 132, 2166 (1985).

${ }^{15}$ E. Gileadi and E. Kirowa-Eisner, Elecrochim. Acta 51, 6003 (2006).

${ }^{16} \mathrm{M}$. Castagnolo and G. Petrella, Electrochim. Acta 19, 855 (1974).

${ }^{17}$ B. R. Boerner and R. G. Bates, J. Sol. Chem. 7, 245 (1978).

${ }^{18}$ T. K. Brownson and F. M. Cray, J. Chem. Soc., Trans. 127, 2923 (1925).

${ }^{19}$ H. Bianchi, H. R. Corti, and R. Fernandez Prini, J. Chem. Soc., Faraday Trans. 1 83, 3027 (1987).

${ }^{20}$ D. L. Goldfarb, M. P. Longinotti, and H. R. Corti, J. Solution Chem. 30, 307 (2001).

${ }^{21}$ Y. C. Wu, W. F. Koch, W. J. Hamer, and R. L. Kay, J. Solution Chem. 16, 985 (1987).

${ }^{22}$ R. M. Fuoss and C. A. Krauss, J. Am. Chem. Soc. 55, 2387 (1933).

${ }^{23}$ R. Fernández Prini, Trans. Faraday Soc. 65, 3311 (1969).

${ }^{24}$ W. M. Haynes, D. Lide, and T. J. Bruno, CRC Handbook of Chemistry and Physics, 93rd ed. (CRC Press, 2012), Section 5-77.

${ }^{25}$ D. L. Goldfarb and H. R. Corti, J. Chem. Phys. B 108, 3358 (2004).

${ }^{26}$ G. Akerlof, J. Am Chem. Soc. 54, 4125 (1932).

${ }^{27}$ U. N. Dash and M. R. Patnaik, Fluid Phase Equilib. 157, 159 (1999).

${ }^{28}$ R. Stairs, Can. J. Chem. 58, 296 (1980).

${ }^{29}$ K. Noda, M. Ohashi, and K. Ishida, J. Chem. Eng. Data 27, 326 (1982).

${ }^{30}$ A. Estrada-Baltazar, A. De Leon-Rodriguez, K. R. Hall, M. RamosEstrada, and G. A. Iglesias-Silva, J. Chem. Eng. Data 48, 1425 (2003).

${ }^{31}$ T. J. F. Day, A. V. Soudackov, M. Cuma, U. W. Schmitt, and G. A. Voth, J. Chem. Phys 117, 5839 (2002); Y. Wu, H. Chen, F. Wang, F. Paesani, and G. A. Voth, J. Phys. Chem. B 112, 467 (2008); K. Park, W. Lin, and F. Paesani, ibid. 116, 343 (2012); C. Knight and G. A. Voth, Acc. Chem. Res. 45, 101 (2012).

${ }^{32}$ A. Warshel and R. M. Weiss, J. Am. Chem. Soc 102, 6218 (1980); A. Warshel, Computer Modelling of Chemical Reactions in Enzymes and Solutions (Wiley, New York, 1991).

${ }^{33}$ M. Born, Z. Phys. 1, 221 (1920).

${ }^{34}$ R. Zanzing, J. Chem. Phys. 52, 3625 (1970).

${ }^{35}$ P. G. Wolynes, Annu. Rev. Phys. Chem. 31, 345 (1980).

${ }^{36}$ B. Bagchi and R. Biswas, Acc. Chem. Res. 31, 181 (1998).

${ }^{37}$ A. K. Srivastava and S. L. Shankar, J. Chem. Eng. Data 45, 92 (2000).

${ }^{38}$ R. M. Fuoss, J. Am. Chem. Soc. 80, 5059 (1958).

${ }^{39}$ F. Wang, S. Izvekov, and G. A. Voth, J. Am. Chem. Soc. 130, 3120 (2008); J. Xu, S. Izvekov, and G. A. Voth, J. Phys. Chem. B 114, 9555 (2010). 